\title{
Prefiguring Floridi's Theory of Semantic Information
}

\author{
John Mingers \\ Kent Business School, Canterbury, UK, j.mingers@kent.ac.uk, \\ http://www.kent.ac.uk/kbs/community/staff/profiles/mingers_john.html
}

\begin{abstract}
Luciano Floridi has been very active in helping to develop both the philosophy of information as a discipline and an actual theory of the nature of semantic information. This paper has three purposes. First, is to demonstrate that Floridi's information theory was largely prefigured by work carried out by Mingers and published some ten years earlier. This is simply a matter of setting the record straight, although the degree of commonality may provide some support for the theory. Second, to point out that there appears to be a degree of equivocation, or even contradiction, within Floridi's theory concerning the ontological status of information - is it objective, independent of the receiver, or is it subjective, constructed by the receiver from the data they access? The paper argues strongly for an objective interpretation. Third, to point out extensions to Mingers' theory in terms of the social and pragmatic aspects of language, the processing of information into meaning through embodied cognition, and the relation between information and different forms of knowledge
\end{abstract}

Keywords: Autopoiesis, Embodied cognition, Information theory, Knowledge, Pragmatics, Semantic information, Theory of communicative action

\section{Introduction}

Luciano Floridi has been hugely influential in both establishing, almost single-handedly, the discipline of information philosophy (Floridi 1999, 2002b, 2003), and constructing a specific theory of semantic information itself (Floridi 2003, 2004b, 2005, 2009b, 2010a) ${ }^{1}$. In this brief note, I wish to discuss the theory of information and, in particular, to draw attention to the fact that much of it was actually prefigured by my own theory of information some ten years before (Mingers 1995, 1996a, 1996b). It is not at all my intention to suggest that Floridi copied my ideas. Rather, as I believe is very common, similar trains of thought and research have occurred relatively independently of each other but have arrived at very similar points. If this is indeed the case, then this triangulation adds strength to the underlying concepts.

In the first section, I will outline my theory of information and in the second I will discuss Floridi's theory, showing how similar it is to my own. Then in the third section I will identify what I consider to be a basic inconsistency in Floridi's approach as evidenced by his writings.

\section{Mingers' Theory of Information and Meaning}

Mingers had essentially the same concern as Floridi, namely to develop a theory of information that would cover its use both semantically and pragmatically. Mingers' analysis began with a semiotic framework due to Morris (Morris 1938) and Stamper (Stamper 1991) that distinguishes four levels at which information can be considered - empirics (the transmission of signs and signals), syntactics (the formal properties of sign systems), semantics (the meaning of signs) and pragmatics (the actual use of signs). The substantive theory was then developed drawing on the work of Dretske (1981), Maturana and Varela (1980) and Habermas (1979). It is cast within a critical realist (Bhaskar, 1978, 1979) framework (Mingers, 2004)2.

\footnotetext{
${ }^{1}$ Floridi's papers have recently been consolidated into a book (Floridi 2011). I will generally refer to the original papers unless there is something substantively different in the book

${ }^{2}$ Floridi (2008a) also supports forms of realism that are very compatible with Bhaskar's critical realism.
} 


\subsection{Analysis of Existing Theories of Information}

The first stage was an evaluation of existing theories of semantic information, at least up to the early 1990s when the work was carried out (Mingers 1996b). At that time a major split could be discerned between those who viewed information as essentially objective, independent of the receiver, and those who viewed it as subjective, constructed by the receiver from the message or sign. The former approach was the standard in applied disciplines such as information systems where information was seen as data that had been processed in some way to make it useful (see Lewis (1991) for a survey). The more radical alternative, proposed by, for example, Checkland $(1990,303)$, was that information was "data plus meaning". That is, observers would receive some data and then apply their own set of meanings to it in order to produce information; different observers generating different information from the same data.

Mingers specified four criteria with which to evaluate the theories:

- Generality - that the theory could cover as wide a range of usages of the term information as possible, in particular that it could account for semantic and pragmatic contexts.

- Relevance - in particular, that it be useful within the disciplines of information systems and information science. This had definitional aspects - that it define clearly the nature of information and distinguish it from related terms such as data and meaning; and behavioural aspects - that it reflected the observed behaviour of information users in real situations, and the interpretive nature of social reality.

- Integration - primarily across disciplines that all use the term information.

- Intuitiveness - if the other criteria are all met then it is better to have a conceptualisation that fits with our intuitive understanding of information rather than one that does not.

A wide range of theories were then examined, many of them developing from Shannon and Weaver's original work on information theory (Shannon and Weaver 1949). Three principal approaches identified were:

- Information as logical possibility. Bar-Hillel and Carnap (Bar-Hillel and Carnap 1952, 1964) developed a system in which the information content of a message within a formal system was measured in terms of the number of other statement in the system that were incompatible with it or, conversely, the number of statement that actually implied it. This has several major limitations: i) it is based on a purely formal system of possible statements and would be quite impractical in a real-world situation. ii) It bases its probabilities on logical possibilities rather than actual occurrences and is better seen as working at the syntactic rather than semantic level. lii) It does not deal at all with the possible interpretations that an observer or receiver may make of a signal or message; it only deals with linguistic propositions, excluding other forms of information.

- Information as reduction in uncertainty. Hintikka (1968), Nauta (1972) and Artandi (1973) all developed theories in which information was related to the reduction in uncertainty brought about by a message in a receiver. This changed the focus to bring in the receiver and their previous state of knowledge and their goals. Thus, if the receiver already knew the content of a message, or if the receiver did not understand the message, it contained no information (for them). This approach was closer to the pragmatic dimension of information, but left the definition of information itself unclear, especially in relation to the difference between meaning and information. It also made information essentially subjective newspapers, timetables and books could not themselves contain information as that only came about when they were consulted.

- Information as a change in cognitive structure. Mackay $(1956,1969)$ also say information as relative to the receiver of a message. In particular, he identified it as the change in cognitive structure (interpreted as a set of readinesses or conditional probabilities) brought about or selected by a particular message. This makes it relative to the prior cognitive state. He also tied the concept explicitly to that of "meaning", recognising three forms - the 
meaning the sender intends, the meaning generated in the receiver, and the "conventional" meaning of the message. The "information content" of a message is then the size or extent of the change brought about in the receiver by the meaning they interpret. Some implications of this theory are: i) two different messages may generate the same meaning for someone, and thus the same information. ii) A message repeated cannot generate any information for the receiver even though it still has meaning. And, iii) a message may not generate any meaning (or information) if it does not have a selective function for the receiver, e.g., if it is in an unknown language.

This theory was made more rigorous by Luhmann (1990), a German phenomenologist, and can be seen as a good model for Checkland's information = data + meaning. However, it still makes information essentially subjective as in the previous approach.

\subsection{Theory of Semantic Information}

Mingers' theory begins from the position that the foundation of information, data and more generally signs, must be differences in the physical world, as Bateson (1973) argued, for without difference there is only uniformity or the void (Spencer-Brown 1972). More particularly, differences that "make a difference", that is generate an event or a sign. Following Dretske (1981) it was argued that events carry information because the occurrence of an event reduces the possibilities of what might happen to what actually does happen, as Shannon and Weaver argued (Shannon and Weaver 1949). In particular, an event (which includes a signal or message) carries the information about what caused it, or led to it. That is, what must be the case in the world for the event to have occurred? Such information exists independently of any observer, indeed, it might never actually be observed. Nevertheless it carries with it the information concerning its own genesis.

Information can also be transmitted provided that there are causal links between the sender (not necessarily a person) and the receiver. This occurs to the extent to which states of the sender are correlated or connected to states of the receiver. Independent events transmit no information; completely linked events transmit all information. Most situations are between the two extremes - the receiver can be affected by things other than the sender (noise), and not all of the information from the source will affect the receive (equivocation). An instrument is a good example - states of the environment, e.g., temperature, causally affect states of the thermometer and the thermometer thus carries information about the temperature. However, things other than the temperature such as magnetism could affect the thermometer, or there could be temperatures outside the range of the thermometer which cannot be registered

Note also that, following Bhaskar (1993), absences can be causes and therefore can generate information. So the gas bill that is not paid by the due date generates information to that effect for the company, which then triggers a reminder letter.

Information is, then, clearly defined - semantic informational is the propositional content of a sign, that is, what is implied about states of affairs in the world given that the sign exists. This definition has several consequences:

- Information is an objective commodity - it is carried by events and signs whether or not it is observed or extracted, and information can be stored and transmitted by the environment, artefacts and people.

- Information is distinct from its embodiment in a sign or message since the information itself can have causal events - a knock on the door leads us to open it not because of the physical knock, but because it carries the information that someone is there. Information itself is therefore not physical but the data that represents or carries it must at some point be physically embodied.

- The amount of information that is available to a particular receiver depends on their prior state (often knowledge) relative to the sign. A book in Chinese has no available information 
for someone who does not read Chinese. Signs about a car not starting will have much more information available for a mechanic than someone who is only a driver. This does not contradict the idea that information is objective - if someone does not know the combination to a safe the money inside is not available to them but still exists.

- A sign carries the information that a particular state of affairs exists, and also all the consequences of that state of affairs that follow nomically, logically, or conventionally.

- Information must be true. We may misunderstand or misinterpret a sign but the sign itself only carries true information. Consider this example - you are looking for a men's toilet and see a picture of a man on a door. You go in and are embarrassed to find it is actually the women's toilet. Does the sign carry information? It clearly does not carry the information that this is the men's toilet, and thus misinformation is not information. It does, however, carry some information, namely concerning why it came to be there. Was it a mistake by the builders, or a practical joke? As observers, we cannot tell because the sign is mute, but it, nevertheless, carries this information and not other, misinformation.

\subsection{Information and Meaning}

The previous section described a theory of semantic information based largely on Dretske. This section describes Mingers' extensions to examine the relationship between information and meaning. To start, it is useful to define some terms:

- Signification. A sign is caused by an event and carries that information. When it is taken, by an observer, as a sign of the event then it is said to have "signification". The sign signifies the causal event. This is essentially semantic information.

- Import. Such a sign will have certain implications for the observer and may lead to some form of action. This is said to be the "import" of the sign for the particular observer.

- Connotation. Signs are direct effects, e.g, a paw print in the ground. Symbols rely on some form of agreed conventions governing their use and meaning (syntax and semantics), e.g., that blue on a map represents water. This is a system of connotations.

- Intent. Finally, in the case of deliberate utterances (which could be linguistic of symbolic), the sender will have some intention in creating the utterance.

These terms allow us to define clearly ambiguous concepts such as data, information and meaning.

- Data is a collection of signs, usually brought together for some purpose, to store or transmit information. They are usually numeric, pictorial or linguistic.

- Semantic information is the propositional content of data, typically in the form of a message but also in the form of a naturally occurring sign.

- Meaning has two different usages. First there is the system of meanings that are publically available within a sign system such as language. These can be drawn on by competent language users in their communications (Habermas 1979). It is that which allows an utterance to carry information but it is not identical to that information. This is termed "connotation" above. The second usage is the "meaning" that the recipient gains from an utterance ("import") and/or that which the sender intends ("intent"). Again, these are all different from the information itself ("signification").

To summarise, let us consider an example - the colour blue on a map which, according to the conventions, connotes that there is water at that place in the world. If there is indeed water there then the map also carries that information. If there actually is not, perhaps be- 
cause of a printer's error, then it does not carry that information. The reader of the map converts the information into meaning (import) in a process that Dretske terms "digitalising the analogue" (Dretske 1981). All signs are ultimately transmitted as a set of analogue differences (light, sound, heat etc) and these get converted into a specific digital distinction by the nervous system. The nervous system extracts from the information available a specific meaning relative to its own prior state, e.g., knowledge and intentions.

Mingers (1995) suggests that this can be seen analytically as happening in three stages: understanding, which involves capturing the basic semantic information carried by the sign or utterance; connotation, at which stage other relevant knowledge that the receiver may have, and the further implications of the information, are brought together; and intent, at which stage the consequences for the receiver are fully realised leading perhaps to a reply, an action, or simply a memory.

The second stage is particularly important in connection with Floridi's theory. The knowledge that it brought into the interpretation of the information is not just individual but socially structured. That is, particular groups will have more or less knowledge, and therefore be able to extract more or less meaning, from a particular source of information, e.g., geographers from maps, and mechanics from cars. These different readinesses to be able to interpret information, or frames of meaning, can be likened to Wittgenstein's forms of life (Wittgenstein, 1958). Note that the transformation of information into meaning is intentional, in a phenomenological sense - it requires a sentient being. Computers can transmit information but cannot transform it into meaning. Conversely, human beings only process meaning, not information.

\subsection{Developments beyond Semantic Information}

In this section I would just like to mention three further developments in my theory of information which go beyond that discussed by Floridi.

The first development is the extension from natural, environmental information to full linguistic utterances (Mingers 1995). This draws on Habermas's theory of communicative action (Habermas 1979; Habermas 1984; Habermas 1987). Put briefly, the information carried by non-linguistic signs (e.g., tree rings or knocks on the door) relates only to its origin, i.e, to what (generally physically) caused it. However, in full human communication there are several extra dimensions that need to be considered in questioning what led to a particular utterance being made. Habermas argues that an utterance implicitly makes four "validity claims", that is basic assumptions that could potentially be challenged. These are, that the utterance is: comprehensible (i.e., well-formed in the language and therefore able to be understood); that it is true with regards to states of affairs in the material world; that it is right in terms of the norms of the social world; and that it is truthful or sincere in terms of the speaker's intentions.

The second development is to give greater consideration to the neurophysiological and cognitive processes that occur in the translation or transformation of information into meaning. Here, I (Mingers 2001) have drawn on the work of phenomenologist Merleau-Ponty (1962; 1963) and biologists Maturana and Varela (Maturana and Varela 1980; Maturana 1978; Varela 1991) and their concepts of autopoiesis and embodied cognition. The essence of this position is to deny the Cartesian split between mind and body, which has been so fundamental in disciplines such as artificial intelligence, computing, information and cognitivist psychology, in favour of one that recognises the essentially embodied nature of human cognition whether at the level of perception, thought, behaviour or language.

The third development is to consider in more detail the relationship between information and knowledge (Mingers 2008). Within information systems it is common to talk of an information hierarchy (Tuomi 1999) from data to information to knowledge although the problem is always to distinguish clearly the differences between the terms and the relationships between them. The theories of information discussed here have largely concerned the relation between data and information, but Dretske saw that information could also generate knowledge. If knowledge is taken to be true, justified belief, as it commonly is, then information, 
which must be true to be information, both generates the belief and also provides the justification to believe it, and therefore generates knowledge for the receiver. Knowledge that " $x$ " is in fact the case. The relationship between information and knowledge has also been considered by Floridi (2004a, 2006, 2010b) but is beyond the scope of this paper.

\section{Floridi's Theory of Semantic Information}

\subsection{Information as Meaningful Data}

Floridi begins with the recognition, following Weaver, that there are different levels at which we can consider the nature of information - the technical aspects concerned with the quantification of information and its transmission, which was dealt with by Shannon and Weaver; the semantic aspect which deals with the meaning of informational messages (and is Floridi's main concern); and the "influential" aspect which concerns the effects of information on human behaviour. This typology is very similar to the one I used, developed by Morris and Stamper, although it has only three levels.

Floridi then asserts that the most common form of an account of information (especially in applied disciplines such as information systems (IS)) is the "bipartite" one in which information is said to be the result of "data + meaning". That is, it consists of a collection of data that is in some sense "meaningful". Floridi $(2005,353)$ does actually quote a paper of mine in support of this view (Mingers 1997). Whilst it is true that I argued that the "data + meaning" account had become common (not the most common), it is not the case that I actually support it, as might be inferred from Floridi's paper.

In fact, at this point I believe Floridi conflates two different views of information - the objective and subjective ones that I discussed above. In his 2005 paper (Floridi 2005), Floridi terms this general theory of information the DOS account, where DOS stands for Declarative, Objective, and Semantic (note the term objective). He then says "Over the last three decades most analyses have supported a definition of DOS information in terms of data + meaning" (p. 353) and gives thee quotations to support this.

"Information is data that has been processed into a form that is meaningful to the recipient" (Davis and Olson 1985, 200)

"Data is the raw material that is processed and refined to generate information" (Silver and Silver 1989, 6)

"Information equals data plus meaning" (Checkland and Scholes 1990, 303)

These three quotes do not, in fact, support the same conceptualisation - there is a major ontological break between the first two and the third. The traditional view of information within IS is that it was data that had been processed in some way to make it more useful (or meaningful) but it remained objective, essentially independent of the receiver. Checkland's work on "soft systems" represented a major break away from an objective view of systems and information towards a subjective, interpretive, phenomenological one (Checkland and Holwell 1998; Lewis 1993). After a similar review of definitions in the existing IS literature, Checkland states:

"The most important feature of this analysis of data, capta, information and knowledge is that the act of creating information is a human act, not one which a machine can accomplish. It is the human being who can attribute meaning to the selected data ... in a context which may well be shared by many people but may also be unique to an individual" (Checkland and Holwell 1998, 91), and "Most of \{the definitions\} do not cover the clear possibility that different people may attribute different meanings to the same data" (Checkland and Holwell 1998, 95).

Thus, for Checkland information is always tied to the receiver(s) who attribute their meanings to the data. The implication is that computers cannot store or transmit information, and books cannot hold information. 
It is interesting that, in the 2009 paper, Floridi (2009b) changes terminology and characterises this bipartite theory as a "general definition of information" (GDI) rather than DOS. In both cases, he analyses it (basing this partly on Devlin's theory (Devlin 1991)) in terms of three conditions:

$\sigma$ is an instance of information, understood as semantic content, iff

$\sigma$ consists of data;

the data are well-formed syntactically (wfd);

the wfd are meaningful.

Thus, information consists of data; the data must conform to some syntactical rules; and the data must involve the "meanings" of the system or recipient. Note that Floridi explicitly accepts that data and information need not be linguistic but could be, for example, pictorial, again inherent in my (and Dretske's) theory.

In fleshing out the GDI theory, Floridi next explicates the nature of data, which is clearly fundamental to GDI (I do not think that it is the case that the various proponents of the bipartite approach (e.g. Checkland 1998) would actually accept all of Floridi's formulation, especially "genetic neutrality" as discussed below). Data rests ultimately on some kind of "difference", that is a lack of uniformity. Without a difference there can be no distinction as Bateson recognised (and Floridi references). Floridi says that the difference may be simply discernable in the physical world (de re); it may be between (perceptions) of different physical states or quantities (de signo); or it may be between different symbols (de dicto). It is important to recognise that data can be decoupled from its physical manifestation (e.g., the number 2 could be represented by two stones or two lines), and the (semantic) information can be decoupled from a particular dataset (e.g., it could be displayed in a picture or writing). Again, this is very much the starting point for my formulation.

Floridi then argues that this conceptualisation implies several degrees of neutrality or underdetermination of the nature of data:

- Taxonomic neutrality (TAXN): data is inherently a relational concept - it is a difference between two relata, but there is no precedence between the relata.

- Typological neutrality (TN): there can be different types of data, e.g., primary data, secondary data, metadata, but whatever type, there can be no information without data. Note however that Floridi allows that an absence may itself be an item of data - the lack of payment of a bill, or answer to a question - may carry information.

- Ontological neutrality (ON): data, and thus information, implies that there must be some form of representation, but it does not have to be a physical one. It could be informationtheoretic, i.e., taking information to be a fundamentally different category to either matter or energy. At some point, however, there must be some form of physical manifestation or representation: "The dependence of information on the occurrence of syntactically wellformed data, and of data on the occurrence of differences variously implementable physically, explains why information can so easily be decoupled from its support. (Floridi, 2009b, p.18, my emphasis).

- Genetic neutrality (GN): by this, Floridi means that the data can come to have a meaning (and therefore be semantic information) independently of any informee (i.e., recipient). The example he gives is the Rosetta Stone which was seen as containing information even before anyone could understand it.

Now this, I believe, would be very controversial among those who put forward the bipartite view. Certainly Checkland (1990) would argue, as we saw above, that the whole point of the "data + meaning" formulation is to show that whereas data may be independent of the informee, information is not. It is precisely the subjective meaning that the individual ascribes or attributes to the data which generates information for them. For example, a clock may read "3.30" (data): one observer may generate the information "I am late for my meeting", 
and another "it's time for tea". It also seems to be in contradiction to Floridi's own ideas later on (Section 3.3) in terms of levels of abstraction (LoA). This marks a major dichotomy between seeing information as objective or subjective.

Floridi draws another distinction here, which is whether information can also be independent of a (human) producer. Some, e.g., Dretske (1981) and myself, would allow environmental information such as tree rings, or animal prints provided that there was some sort of causal link which generated, and perhaps transmitted, the information. Note that Floridi maintains that environmental information may well be used, by animals and plants, but not be "meaningful" if it is not processed by human agents. Floridi also makes the point (Floridi $2009 \mathrm{~b}, 21$ ) that the absence of data, as in the lack of answer to a question, may itself generate information, again a similarity with Mingers.

What is Floridi's own evaluation of GDI (or DOS)? In Floridi (2005) he argues that the DOS definition provides necessary but not sufficient conditions for data to become information, and that what is additionally needed is the stipulation that the data is also truthful, as will be shown below.

\subsection{The Mathematical Theory of Communication}

Having developed the GDI as a background to other information theories, Floridi next explains Shannon and Weaver's (1949) mathematical theory of communication. This is generally accepted as the most rigorous and well-developed analysis of some properties of information, or at least signal transmission, but is clearly not applicable at the level of semantics or meaning. It is better seen as a theory of data transmission and has nothing to say about the actual content of a message. However, he then points out that the fundamental ideas have been used as the basis for several well-known theories of semantic information including Bar-Hillel and Carnap (1952), Mackay (1969), and Nauta (1972), all of whom were reviewed in my paper.

\subsection{Factual Information}

Floridi now develops his own view on information and, in particular, the necessary relationship with truth and then with knowledge. For Floridi, information is the true semantic content of data. Again, this is just what I (and Dretske) argued and makes the theory different from most other information theories. There are three important steps in his argument.

First, data describe states of affairs in the world and may be true or false. Such data generates affordances or constraints for the agent who receives (and understands) it. For example, if the car stops and the petrol gauge reads empty this data makes it more likely that the agent comes to believe the car has run out of petrol (affordance) and less likely they will think the battery is flat (constraint). At this point we reach a significant step in the theory - Floridi says that it is the agent or observer who "constructs the information" from the data (Floridi $2009 b, 36)$. This is significant for the ontology of information for essentially it makes it subjective rather than objective. It is data that exists outside of the observer or receiver, but the information only comes into existence with the processing or interpretation of the data by a person (or perhaps other sentient being). This would seem to place Floridi in the same camp as Checkland and those who argue that information is data + meaning.

The second step is the process by which data is transformed into meaning. Here, Floridi brings in a concept that he calls "levels of abstraction" (LoA) (Floridi 2008b). This is essentially an interface between the data and the receiver. It is epistemological rather than ontological. A specific LoA specifies the type of data that can be processed by a receiver if that data is available. In more usual terms it could be said to be the specific set of relevant constructs or system of meanings that is available to the particular observer. Floridi recognises that this may not be purely individual - different types of people - e.g., drivers, engineers or economists - may bring with them different LoAs to a particular set of data, e.g., that car not working, and thereby construct or generate different information from the same data.

This idea is very similar to my analysis of the way that information is transformed into meaning, namely that the information available to a particular receiver will depend on the 
personal knowledge that the receiver has, and also the knowledge they have by virtue of being a particular type of person.

The third step is to consider the question of the truthfulness of information. For some theorists, such as Devlin (1991), Colburn (2000) and Fetzer (2004), meaningful, well-formed data constitutes information whether or not it is actually true. However, Floridi sides with those such as Dretske (1981) and Grice (1989) who argue that false information, misinformation or disinformation is not actually information at all. I will not repeat the debate here as my theory accepts it as well but see Floridi (2004b, 2005) for the detail.

\subsection{Quantifying Semantic Information}

Finally, Floridi considers the question of whether semantic information can be quantified (Floridi, 2004b). There have been several approaches to this problem. For example, the mathematical theory of communication which, as we have seen, does not apply to semantic information; and Bar-Hillel and Carnap (1952) who base their method on Shannon and Weaver but calculate the amount of information that a message or statement carries in terms of the number of possibilities it logically excludes within the set of all possible messages in a pre-defined system of terms.

My own view is that it is inappropriate to try and quantify information at the semantic or pragmatic levels. The principal argument is very simple - proper quantification (of the sort implied by Floridi's (2009b) Figure 5) requires that the constructs to be quantified can be measured on an interval scale of measurement but much semantic content, especially of a linguistic kind, can only be measured on nominal or ordinal scales. One can understand, and perhaps compare, meanings but one cannot generally measure them, or measure their degree of divergence from one another.

\section{Comparison and Evaluation - Is Information Objective?}

I believe that I have demonstrated a significant number of similarities in the two theories. From their origins in difference and distinction and their rejection of theories based on the mathematical theory of communication, through to the idea of semantic information being data that represents true states of affairs in the world, and the similarity between Floridi's levels of abstraction (LoA) and my frames of meaning. As I said in the introduction, the point of this is simply to establish the extent to which Floridi's theory was prefigured by my own in order to set the record straight.

However, there also appears to be a significant difference in the endpoint, perhaps generated by potential contradictions in Floridi's approach. The issue concerns the exact ontological status of information - is it objective, existing independently of any receiver or (in the case of environmental information) producer, as Dretske and myself would argue? Or, is it subjective, constructed anew by the receiver when they interact with data, as Checkland would hold? This is clearly a fundamental question for any theory of information. To be clear, we can label three different positions:

- OBJ1: The received view within information systems is that information is objective as it is just data that has been processed in some way. It is then transferred unproblematically into the cognition of observers.

- SUBJ: Here, following Checkland, MacKay and Luhmann, information is some change in state of the cognitive apparatus of an observer. It is therefore intrinsically subjective. Data is interpreted through the observer's system of meaning to generate information for the observer.

- OBJ2: Dretske and Mingers argue that data carries information which is therefore objective, potentially able to be accessed by observers. This generates meaning, and potentially knowledge, for the observer through a process of embodied cognition.

Now, the question is where does Floridi's conception of semantic information stand with respect to these three possibilities? The answer, it seems to me, is somewhat equivocal in 
his various writings. It is quite possible to make out a case, especially from the earlier writings, that semantic information is objective, independent of the observer. But it is also possible to see passages which suggest that information is indeed, at least in part, dependent on the observer or receiver, and therefore not wholly objective in the sense of OBJ2. We will develop these two competing viewpoints.

In the 2005 paper, as we have seen, Floridi accepts the DOS view of information, including its claim to objectivity, arguing only that it needs to be extended to include the stipulation that information must be true to be information. However, by 2009 the theory is called GDI (General Definition of Information), perhaps significantly excluding the connotation of objectivity. Nevertheless, the criterion of genetic neutrality $(\mathrm{GeN})$ states that data can have a semantics (i.e., it can be meaningful) independently of an informee. Whilst this is not quite the same as saying that the actual information is independent, Floridi does go on to say:

"GeN supports the possibility of information without an informed subject ... Meaning is not (at least not only) in the mind of the user. GeN is to be distinguished from the stronger, realist thesis, supported by Dretske (1981), according to which data could also have their own semantics independently of an intelligent produced/informer" (Floridi 2005, 23)

This latter point is the slightly different one, namely can information be produced unintentionally? For example, does a paw print in the jungle carry information about the presence of an animal - is it data that is well-formed and meaningful? Dretske (and myself) would say yes; Floridi is not sure that such data can in fact be meaningful.

In Floridi (2009a), semantic information is redefined as "p qualifies as semantic information if and only if $p$ is (constituted by) well-formed, meaningful and veridical data" (Floridi 2009a, 143). Then it is stated that "a large variety of kinds of semantic information, from traffic lights to train timetables, from road signs to fire alarms, falls within the scope fof this definition\}" (Floridi 2009a, 146). It may seem rather strange to categorise a physical object such as a traffic light, in itself, as an instance of well-formed, veridical data (rather than, say, simply a generator of such data), a point we will return to later in the paper. But, for our purposes it does at least show that Floridi appears happy with the idea that there are examples of semantic information that are external to and independent of an observer, and thus corresponding to OBJ2.

Going further, Floridi (2008a) has developed a metaphysics based around the idea of informational objects (the "infosphere") that he calls "informational structural realism" (ISR). He summarises this as follows:

"As a form of realism, ISR is committed to the existence of a mind-independent reality addressed by and constraining our knowledge. ... A significant consequence of ISR is that, as far as we can tell, the ultimate nature of reality is informational, that is it makes sense to adopt LoA s that commit out theories to a view of reality as mind-independent and constituted by structural objects that are neither substantial nor material but informational." (Floridi 2008a, 240)

And,

"A straightforward way of making sense of these structural objects is as informational objects, that is cohering clusters of data, not in the alphanumeric sense of the word, but in an equally common sense of differences de re, i.e., mind-independent, concrete points of lack of uniformity" (Floridi 2008a, 236)

So, all of the above seems to suggest that Floridi has a strong sense of information being objective, both epistemologically and ontologically.

However, in places things seem less clear. We read that: 
"The data that constitute factual information allow or invite certain constructs (they are affordances for the information agent that (sic) can take advantage of them) and resist or impede some others (they are constraints for the same agent) depending on the interaction with, and the nature of, the information agent that processes them. For example, the red light flashing repetitively and the engine not starting allow you (or any other information agent like you) to construct the information ... This is the sense in which data are constraining affordances for (an information agent responsible for) the elaboration of factual information" (Floridi 2009b, 36 orig. emphasis)

"\{LoAs\} are interfaces that mediate the epistemic relation between the observed and the observer. ... Data ... are translated into factual information by being processed semantically at a given LoA" (Floridi 2009b, 37)

The picture that seems to emerge from these quotes is very much the subjectivist one in which objective (i.e., external) data is processed by a receiver applying their meaning system (LoA) to it in such a way as to generate or construct information. Factual information only comes into existence when data has been processed by an "information agent". In fact, Floridi labels his approach as "constructionist" (as opposed to "constructivist"):

"From this perspective, the world is neither discovered nor invented but designed by the epistemic agents experiencing it. This is neither a realist nor an anti-realist but a constructionist view of information" (Floridi 2011, 78)

There does seem to me to be a distinct difference between the two position on information outlined above. In the first, semantic information is identified with data, albeit only a special type of data (well-formed, meaningful, true), it actually is nothing but the data. In the second case, information appears to be different to but derivable from data with suitable processing by an agent. In the first case, information is objective, in the second it is at least partly subjective, driven both by the affordances/constraints of the data, and the knowledge and purposes of the agent. Apart from the fact that this is a substantive inconsistency within Floridi's overall theory, I would argue that in fact both positions are problematic.

Consider the following examples with regard to the first position:

Ex1: "gsyn tthh5 kounf gttre"

Ex2: "Today is Thursday $11^{\text {th" }}$

Ex3: "Hooray"

Ex1 is data but cannot be information because it is not well-formed or meaningful. Ex2 is well-formed and meaningful but is only information if it is in fact true that the day on which I am writing this is in fact Thursday $11^{\text {th }}$ (which it is). Ex3 is well-formed and meaningful but I do not think that it can be either true or false, it is simply an exclamation. In order to be true, something must bear some relationship to something else against which its truth or falsity can be assessed. These examples show that it is problematic to identify information purely with data, even of a special type. It is much better to say that information is "carried by" or "represented in" data but is separate to it. This allows for the proper degrees of neutrality of information from its particular representation. In Dretske and my versions, information is "the propositional content of a sign", it is not the sign itself.

In terms of the second position, it leads to a confusion between meaning and information. When the observer reacts to some data it generates a cognitive change in them. Is this change meaning or information? Either the two are in fact referring to the same thing in which case one is redundant; or they are different but then what exactly is the difference?

From my perspective, data, both linguistic and natural, carry information about states of affairs in the world. This is objective and independent and exists whether or not anyone actually interacts with it. Receivers then process this information, given the existing state of their nervous system, in such a way as to produce meaning (import) for them. Thus, information is objective and meaning is subjective. This makes it perfectly reasonable to say that machines and artefacts can store and perhaps transmit information while people generate 
individual interpretations of it. This also fits better with the stipulation that information must be true. When a receiver, through a LoA, processes data into information they may always be mistaken in which case the result would not actually be information. There is no such problem with meaning, for meaning does not have to be true.

\section{Conclusions}

This paper has made two contributions. First, that Floridi's theory of information was largely prefigured by that of Mingers, which itself was a synthesis of a range of theorists including Dretske, Maturana and Varela, Bhaskar and Habermas. The point of this is simply to set the record straight in terms of the origin of theoretical ideas. To the extent that the two theories are similar, then this provides some corroboration of the underlying ideas.

Second, that there appears to be a degree of equivocation, if not actual inconsistency, about the ontological status of information within Floridi's theory. In earlier papers he seemed to support a DOS view of information which made it clearly objective, independent of the receiver of a message. However, in later papers it appears that information is generated or constructed by the receiver upon receipt of data, which would make it subjective. Obviously this is a vital question that needs to be answered in as clear a manner as possible. Mingers (and Dretske) both support the view that information is objective.

Looking to the future, "information" is obviously a fundamental, transdisciplinary concept, and it is somewhat of a scandal that there is not yet an agreed and accepted definition. It would be a major advance if such a concept could emerge, and Floridi's work in developing both a philosophy of information and theory of information is playing a major role in this. It must be acknowledged that he has developed his own ideas in a major way towards the concept of the "infosphere" which envisages reality as consisting of informational structures or objects that have their own intrinsic properties including ethical rights (Floridi 2002a, 2008a)

\section{References}

Artandi, Susan 1973. Information Concepts and Their Utility. Journal of the American Society for Information Science 24 (4): 242-245.

Bar-Hillel, Yehoshua and Rudolf Carnap. 1952. An Outline of a Theory of Semantic Information. Cambridge: Research Laboratory of Electronics, MIT.

Bar-Hillel, Yehoshua and Rudolf Carnap. 1964. Language and Information: Selected Essays on their Theory and Application. Reading: Addison-Wesley.

Bateson, Gregory. 1973. Form, Substance and Difference. In Steps to an Ecology of Mind, edited by Gregory Bateson, 423-440. London: Paladin.

Bhaskar, Roy. 1978. A Realist Theory of Science. Hemel Hempstead: Harvester.

Bhaskar, Roy. 1979. The Possibility of Naturalism. Sussex: Harvester Press.

Bhaskar, Roy. 1993. Dialectic: the Pulse of Freedom. London: Verso.

Checkland, Peter and Sue Holwell. 1998. Information, Systems and Information Systems: Making Sense of the Field. Chichester: Wiley.

Checkland, Peter and Jim Scholes. 1990. Soft Systems Methodology in Action. Chichester: Wiley.

Colburn, Timothy. 2000. Philosophy and Computer Science. Armonk: M.E. Sharpe.

Davis, Gordon and Margrehte Olson. 1985.. Management Information Systems: Conceptual Foundations, Structure and Development. New York: McGraw Hill.

Devlin, Keith. 1991. Logic and Information. Cambridge: Cambridge University Press.

Dretske, Fred. 1981. Knowledge and the Flow of Information. Oxford: Blackwell.

Fetzer, James. 2004. Information: Does it Have to Be True? Minds and Machines, 14 (2), 223-229.

Floridi, Luciano. 1999. Information Ethics: On the Philosophical Foundation of Computer Ethics. Ethics and Information Technology 1(1): 37-56.

Floridi, Luciano. 2002a. On the Intrinsic Value of Information Objects and the Infosphere. Ethics and Information Technology 4: 287-304.

Floridi, Luciano. 2002b. What is the Philosophy of Information? Metaphilosophy 33: 123-145.

Floridi, Luciano. 2003. Two Approaches to the Philosophy of Information. Minds and Machines 13 (4): 459-469. 
Floridi, Luciano. 2004a. On the Logical Unsolvability of the Gettier Problem. Synthese 142 (1): 61-79.

Floridi, Luciano. 2004b. Outline of a Theory of Strongly Semantic Information. Minds and Machines 14 (2): 197-221.

Floridi, Luciano. 2005. Is Semantic Information Meaningful Data? Philosophy and Phenomenological Research 70 (2): 351-370.

Floridi, Luciano. 2006. The Logic of Being Informed. Logique et Analyse 49 (196): 433-460.

Floridi, Luciano. 2008a. A Defence of Informational Structural Realism. Synthese 161 (2), 219-253.

Floridi, Luciano. 2008b. The Method of Levels of Abstraction. Minds and Machines 18 (3), 303-329.

Floridi, Luciano. 2009a. Outline of a Theory of Truth as Correctness for Semantic Information. tripleC 7 (2): 142-157.

Floridi, Luciano. 2009b. Philosophical Conceptions of Information. In Formal Theories of information edited by Giovanni Sommaruga, 13-53. Berlin: Springer-Verlag.

Floridi, Luciano. 2010a. Information, Possible Worlds, and the Cooptation of Scepticism. Synthese 175 (1): 63-88.

Floridi, Luciano. 2010b. Semantic information and the correctness theory of truth. Erkenntnis 72: 147175.

Floridi, Luciano. 2011. The Philosophy of Information. Oxford: Oxford University Press.

Grice, H. Paul. 1989. Studies in the Way of Words. Cambridge: Harvard University Press.

Habermas, Jürgen. 1979. Communication and the Evolution of Society. London: Heinemann.

Hintikka, Jaakko. 1968. The Varieties of Information and Scientific Explanation. In Logic, Methodology and Philosophy of Science III, edited by Bob van Rootselar and Frits Staal, 311-331. Amsterdam: North Holland.

Lewis, Paul. 1991. The Decision-Making Basis for Information Systems: The Contribution of Vickers' Concept of Appreciation to a Soft Systems Perspective. European Journal Information Systems 1 (1): 33-43.

Lewis, Paul. 1993. Identifying Cognitive Categories - The Basis for Interpretative Data-Analysis Within Soft Systems Methodology. International Journal of Information Management 13 (5): 373-386.

Luhmann, Nicholas. 1990. Meaning as Sociology's Basic Concept. In Essays in Self-Reference, edited by Nicholas Luhmann, 21-79. New York: Columbia University Press.

Mackay, Donald. 1956. The place of "meaning" in the theory of information. In Information Theory: Third London Symposium, edited by ColinCherry, 215-225. London: Butterworth.

Mackay, Donald. 1969. Information, Mechanism and Meaning. Cambridge MA: MIT Press.

Maturana, Humbert and Francisco Varela. 1980. Autopoiesis: The Organization of the Living. In Autopoiesis and Cognition: The Realization of the Living, edited by Humbert R. Maturana and Francisco J. Varela, 63-134. Dordrecht: Reidel.

Mingers, John.1995. Information and meaning: foundations for an intersubjective account. Information Systems Journal 5 (4): 285-306.

Mingers, John. 1996a. Embodying Information Systems. In Information Technology and Changes in Organizational Work edited by Wanda Orlikowski, Geoff Walsham, Matthew Jones, and Janice DeGross, 272-292, London: Chapman Hall.

Mingers, John. 1996b. An Evaluation of Theories of Information with Regard to the Semantic and Pragmatic Aspects of Information Systems. Systems Practice 9(3): 187-209.

Mingers, John. 1997. The Nature of Information and its Relationship to Meaning. In Philosophical Aspects of Information Systems, edited by Russel Winder, Stephen Probert and lan Beeson, 7384. London: Taylor and Francis.

Mingers, John. 2004. Re-Establishing the Real: Critical Realism and Information Systems Research. In Social Theory and Philosophy for Information Systems edited by John Mingers and Leslie P. Willcocks, 372-406. London: Wiley.

Morris, Charles. 1938. Foundations of the Theory of Signs. In International Encyclopedia of Unified Science Vol 1 edited by Otto Neurath. Chicago: University of Chicago Press.

Nauta, Doede. 1972. The Meaning of Information. The Hague: Mouton.

Shannon, Claude and Warren Weaver. 1949. The Mathematical Theory of Communication. Illinois: University of Illinois Press.

Silver, Gerald and Myrna Silver. 1989. Systems Analysis and Design. Reading: Addison-Wesley.

Spencer-Brown, George. 1972. Laws of Form. New York: Julien Press.

Stamper, R. 1991. The Semiotic Framework for Information Systems Research. In Information Systems Research: Contemporary Approaches and Emergent Traditions, edited by Hans-Erik Nissen, Heinz Klein and Rudy Hirscheim, 515-528. Amsterdam: North Holland. 
Wittgenstein, Ludwig. 1958. Philosophical Investigations. Oxford: Blackwell.

\section{About the Author}

\section{John Mingers}

is Professor of Operational Research and Systems at Kent Business School, and Director of the School. Prior to this, he was at Warwick Business School. His research interests include the development of theory concerning the nature of information, meaning and knowledge; the use of systems methodologies in problem situations - particularly the mixing of different methods within an intervention (multimethodology); the development of critical realism as an underpinning philosophy for information systems; research metrics, and autopoiesis and its applications. He is an Editor of MIS Quarterly 